Originally published as:

Bettina Rosner, Helen Bernard, Dirk Werber, Mirko Faber, Klaus Stark, Gérard Krause. Epidemiologie des EHEC 0104:H4/HUS-Ausbruchs in Deutschland, Mai bis Juli 2011.

(2011) Journal für Verbraucherschutz und Lebensmittelsicherheit, 6 (4), pp. 473-481.

DOI: DOI 10.1007/s00003-011-0740-1

This is an author manuscript.

The definitive version is available at: http://www.springer.com 


\title{
Epidemiologie des EHEC 0104:H4/HUS- Ausbruchs in Deutschland, Mai bis Juli 2011
}

\author{
Bettina Rosner, Helen Bernard, Dirk Werber, Mirko Faber, Klaus Stark, Gérard Krause
}

\section{Zusammenfassung}

Von Mai bis Juli 2011 ereignete sich vor allem in Norddeutschland der bisher größte Ausbruch von Infektionen durch enterohämorrhagische Escherichia coli (EHEC) 0104:H4 und von hämolytisch-urämischem Syndrom (HUS) mit insgesamt über 3.800 Erkrankten und 53 Todesfällen. Bei den Erkrankten handelte es sich überwiegend um Erwachsene.

Dies steht im Gegensatz zu den in den Meldedaten der letzten Jahre beobachteten Erkrankungsfällen an EHEC-Gastroenteritis und HUS, als vor allem Kinder betroffen waren. Der Ausbruch begann, bezogen auf den Erkrankungsbeginn (Durchfallsymptomatik) der übermittelten Fälle, am 08. Mai 2011, gipfelte am 22. Mai und klang danach ab. Am 26. Juli 2011 wurde der Ausbruch vom Robert Koch-Institut (RKI) für beendet erklärt, da zu diesem Zeitpunkt drei Wochen lang keine neuen, offensichtlich dem Ausbruch zugehörigen Erkrankungsfälle übermittelt

worden waren, die nach dem 4. Juli erkrankt waren. Epidemiologische Studien des RKI in Kooperation mit dem öffentlichen Gesundheitsdienst von Ländern und Kommunen und Kliniken sowie Ermittlungen der Lebensmittelüberwachungsbehörden identifizierten mit großer Wahrscheinlichkeit kontaminierte Sprossen aus einem Gartenbaubetrieb in Niedersachsen bzw. importierte Bockshornkleesamen als Infektionsvehikel im Ausbruchsgeschehen. Die enge Kooperation der Gesundheits- und Lebensmittelüberwachungsbehörden ermöglichte die zeitnahe Aufklärung des Ausbruchsgeschehens.

\section{Einleitung}

Infektionen mit Shigatoxin (Stx)-bildenden Escherichia coli (STEC) können beim Menschen zu hämorrhagischer Colitis und dem lebensbedrohlichen hämolytisch-urämischen Syndrom (HUS) führen. Das klinische Bild eines HUS umfasst akutes Nierenversagen, hämolytische Anämie und Thrombozytopenie. Der Krankheitsverdacht, die Erkrankung und der Tod an enteropathischem HUS sowie der Nachweis von enterohämorrhagischen E. coli (EHEC; hier: Synonym für STEC) sind in Deutschland nach §6 bzw. §7 des Infektionsschutzgesetzes (IfSG) meldepflichtig.

Zwischen 2001 und 2010 wurden jährlich etwa 1.000 symptomatische EHEC-Infektionen und etwa 60 HUS-Fälle an das Robert Koch-Institut (RKI) übermittelt. Betroffen waren vor allem Kinder unter fünf Jahren. Im Jahr 2010 traten zwei Todesfälle im Zusammenhang mit HUS Erkrankungen auf (RKI 2011a).

Ein wichtiges Reservoir für EHEC sind Wiederkäuer, insbesondere Rinder, Schafe und Ziegen. Die Infektion des Menschen erfolgt häufig über die unbeabsichtigte Aufnahme von Fäkalspuren, z. B. über kontaminierte Lebensmittel, Wasser oder den direkten Kontakt zu Tieren, aber auch über direkte übertragungen von Mensch zu Mensch (Schmierinfektionen).

Die minimale Infektionsdosis von EHEC ist sehr gering (\100 Erreger für Serogruppe O157).

EHEC-Krankheitsausbrüche, vor allem Ausbrüche, die durch E. coli O157 hervorgerufen wurden, sind weltweit häufig beschrieben worden. Kontaminierte Lebensmittel sind die häufigste Ursache von EHECAusbrüchen. Sowohl der Verzehr von Lebensmitteln tierischen Ursprungs wie nicht durchgegartem Rinderhackfleisch (Bell et al.1994; King et al. 2009) und Rohmilch (Keene et al. 1997) als auch von Lebensmitteln pflanzlichen Ursprungs, z. B. Blattsalat (Ackers et al. 1998), Rettichsprossen (Michino et al. 1999) und Alfalfasprossen (Breuer et al. 2001), konnten als Infektionsursachen in Ausbruchsgeschehen identifiziert werden.

Andere Ausbrüche wurden dem Kontakt zu Badegewässern (Keene et al. 1994) und Schwimmbecken (Verma et al. 2007) oder dem Besuch von Streichelzoos (CDC 2005) zugeschrieben. 
Von Anfang Mai bis Ende Juli 2011 trat in Deutschland ein großer EHEC-Ausbruch mit E. coli des seltenen Serotyps O104:H4 auf, der letztlich auf Bockshornkleesprossen

bzw. Bockshornkleesprossensamen als Infektionsvehikel zurückgeführt werden konnte. Im

Folgenden werden die Epidemiologie des Ausbruchsgeschehens sowie epidemiologische Studien des

RKI, die zur Identifizierung des Infektionsvehikels beitrugen, zusammenfassend dargestellt.

Dieser Artikel steht in engem Zusammenhang mit dem RKI-Abschlussbericht ,Abschließende

Darstellung und Bewertung der epidemiologischen Erkenntnisse im EHEC 0104:H4

Ausbruch, Deutschland 2011“"

(http://www.rki.de/cln_169/nn_205760/DE/Content/InfAZ/E/EHEC/EHEC-

Abschlussbericht,templateld=raw, property=publicationFile.

pdf/EHEC-Abschlussbericht.pdf) sowie anderen Artikeln zum Leitthema EHEC in diesem Journal, die die Ausbruchsuntersuchungen aus Sicht der Lebensmittelüberwachungsbehörden bzw. der Task Force EHEC schildern.

\section{Deskriptive Epidemiologie des Ausbruchs}

Am 19. Mai 2011 wurde das RKI von der Hamburger Behörde für Gesundheit und Verbraucherschutz über das Auftreten einer Häufung von drei HUS-Erkrankungen bei Kindern informiert und eingeladen, die Behörden vor Ort bei ihren Untersuchungen zu unterstützen.

Ein erstes RKI-Team reiste am 20. Mai nach Hamburg und führte zusammen mit Mitarbeitern der lokalen Behörden eine ausführliche explorative, d. h. hypothesengenerierende, Befragung von Patienten bzw. Eltern von erkrankten Kindern hinsichtlich möglicher Infektionsursachen und -vehikel durch.

Die Zahl der HUS- und EHEC-Erkrankungen in den Hamburger Krankenhäusern stieg schnell an und es wurde sehr bald klar, dass es sich um ein ungewöhnliches Geschehen handelte, unter anderem da vor allem Erwachsene von HUS betroffen waren (Frank et al. 2011).

Daraufhin wurde eine intensive Ausbruchsuntersuchung eingeleitet, die die kommunale, Landes- und Bundesebene des öffentlichen Gesundheitsdienstes und der Lebensmittelüberwachungsbehörden einband.

Im Rahmen der Routine-Surveillance werden HUS und EHEC-Fälle standardisiert erfasst und die Meldungen von den lokalen Gesundheitsbehörden über die Landesstellen an das RKI übermittelt. Im Ausbruchsgeschehen wurden die Gesundheitsämter und Landesstellen gebeten, Meldungen zu HUSErkrankungen und HUS-Verdachtsfällen nach deren Eingang umgehend an das RKI zu übermitteln. Zusätzlich wurde Ende Mai eine syndromische Surveillance von Patienten mit blutigen Durchfällen in Notaufnahmen von freiwillig teilnehmenden Krankenhäusern etabliert (Wadl et al. 2011).

Bis zum 26. Mai 2011 lagen detaillierte mikrobiologische Ergebnisse zum Ausbruchsstamm aus dem Nationalen Referenzzentrum für Salmonellen und andere Enteritiserreger am RKI in Wernigerode und Dem Konsiliarlabor für HUS an der Universität Münster vor.

Charakteristisch für den Sorbitol fermentierenden Ausbruchsstamm vom Serotyp O104:H4 sind Virulenzmerkmale enteroaggregativer E. coli, das Virulenzmarkergen stx2 (für die Bildung von Shigatoxin 2), die Abwesenheit der Virulenzmarker stx1 und eae sowie die Bildung einer ExtendedSpectrum Beta-Laktamase (ESBL positiv) (Bielaszewska et al. 2011).

Für die Zählung und Berichterstattung des RKI zu EHEC / HUS-Fällen wurden dem Ausbruchsgeschehen die HUS-Fälle und EHEC-Infektionen zugerechnet, die die AusbruchsFalldefinition erfüllten (http://www.rki.de/cln_178/nn_467482/DE/Content/InfAZ/E/EHEC/ Falldefinition.html). Eingeschlossen wurden Fälle mit einem Erkrankungsbeginn (typisches Erstsymptom Durchfall) zwischen dem 01. Mai 2011 und dem 04. Juli 2011. Erkrankungsfälle mit unbekanntem Erkrankungsdatum wurden von der 19. Meldewoche (begann am 09. Mai) bis zur 28. Meldewoche (endete am 17. Juli) gezählt. Ausgeschlossen wurden Fälle, bei denen eine andere EHEC-Serogruppe als die des Ausbruchsstammes $\mathrm{O} 104$ nachgewiesen wurde, sowie Fälle mit EHEC-Infektion ohne Serogruppeninformation, die als isoliert stx1-positiv übermittelt wurden. EHECassoziierte HUS-Fälle wurden nicht als EHEC-Fälle gezählt. Dem RKI wurden 855 HUSErkrankungen (einschließlich HUS-Verdachtsfälle) und 2.987 EHEC Infektionen, also insgesamt 3.842 dem Ausbruch zuzurechnende Erkrankungen gemäß IfSG und Ausbruchsfalldefinition übermittelt (Datenstand 16.08.2011; RKI 2011b). Insgesamt 53 Personen verstarben, davon 35 
(4.1\%) HUS-Patienten und 18 (0.6\%) EHEC-Patienten. In Abbildung 1 ist die epidemiologische Kurve der HUS und EHEC-Fälle mit Erkrankungsbeginn (Durchfall) seit dem 01. Mai 2011 dargestellt. Beide Kurven steigen ab dem 08. Mai stark an, gipfeln am 21./22. Mai und fallen dann ab. Der Erkrankungsbeginn der letzten dem Ausbruch zugerechneten Fälle ist der 04. Juli 2011 (Abb. 1). Die nördlichen Bundesländer Hamburg, Schleswig-Holstein, Bremen, MecklenburgVorpommern und Niedersachsen waren mit HUS-Erkrankungsinzidenzen von 1,8 bis 10,0 Fällen pro 100.000 Einwohnern besonders stark vom Ausbruchsgeschehen betroffen.

Fälle wurden aus allen 16 Bundesländern berichtet. Auffällig war, dass sich die große Mehrzahl der Fälle wahrscheinlich in Norddeutschland infiziert hatte (Abb. 2) (Askar et al. 2011).

Nach Angaben des European Centre für Disease Prevention and Control (ECDC) traten in der Europäischen Union außerhalb Deutschlands insgesamt 76 EHEC-Fälle und 49 HUS-Fälle auf, eine Person verstarb an HUS (ECDC 2011; Datenstand 26.07.2011; http://www.ecdc.europa.eu). Erkrankungsfälle wurden auch aus dem außereuropäischen Ausland, z. B. USA, Kanada berichtet (8 EHEC-Fälle, 4 HUS-Fälle, davon ein HUS-Todesfall; WHO 2011, Datenstand 21.07.2011; http://www.euro.who.int). Auch die meisten Fälle, die im Ausland auftraten, waren vor ihrer Erkrankung nach oder durch Norddeutschland gereist. Im Gegensatz zu HUS-Fällen, die dem RKI zwischen 2001 und 2010 übermittelt wurden, waren die HUS Ausbruchsfälle deutlich älter.

Der Altersmedian betrug 42 Jahre (Spanne: 0-91 Jahre), nur etwa $2 \%$ der HUS-Fälle waren jünger als 5 Jahre. Bei den bis 2010 übermittelten HUS-Fällen waren dagegen $69 \%$ jünger als 5 Jahre. Der Altersmedian der HUS-Todesfälle lag bei 74 Jahren (Spanne: 2-91 Jahre). Im Ausbruch waren mehr Frauen $(68 \%)$ als Männer von einem HUS betroffen. Auch bei EHEC-Erkrankungen waren mehr Erwachsene als Kinder betroffen; der Altersmedian betrug 46 Jahre (Spanne: 0-100 Jahre). Der Altersmedian der EHEC-Todesfälle lag bei 83 Jahren (Spanne: 38-89 Jahre). Frauen waren etwas häufiger (58\%) von EHEC-Infektionen betroffen als Männer. Dies entsprach in etwa dem Anteil der weiblichen Personen bei den EHEC-Fällen unter erwachsenen Personen C20 Jahre, die dem RKI zwischen 2001 und 2010 übermittelt wurden (61\%).

Die Inkubationszeit, die für epidemiologische Untersuchungen (z. B. damit bei Befragungen von Patienten der korrekte Expositionszeitraum berücksichtigt wird), die Einschätzung der epidemiologischen Entwicklung und die Festlegung präventiver Maßnahmen von großer Bedeutung ist, war mit einem Median von 8 Tagen (25\% Perzentil: 6 Tage; $75 \%$ Perzentil: 10 Tage) in diesem Ausbruch länger im Vergleich zu Angaben in der Literatur und Erfahrungen bei vorangegangenen Ausbrüchen (Angaben für EHEC 0157: Median 3-4 Tage; Heymann 2008). Die Inkubationszeit im Ausbruchsgeschehen wurde anhand von Angaben von Fällen, für die ein Erkrankungsbeginn und ein vermutlicher Expositionszeitraum von maximal 2 Tagen angegeben worden war, geschätzt. Für die Schätzung wurden Angaben aus dem gesetzlichen Meldesystem, aus Restaurant-Clustern und von ausländischen Fällen berücksichtigt. Die Zeit zwischen Durchfallbeginn und dem Auftreten eines HUS betrug im Median 5 Tage (25\% Perzentil: 4 Tage; $75 \%$ Perzentil: 7 Tage) (RKI 2011b). Damit scheint die Dauer zwischen Durchfallbeginn und Beginn eines HUS bei dem Ausbruchsstamm etwas kürzer zu sein als bei Infektionen mit enterohämorrhagischen E. coli der Serogruppe 0157 (Median 7 Tage; Tarr et al. 2005).

\section{Epidemiologische Studien des Robert Koch-Instituts zur Identifizierung des Infektions- vehikels}

Seit dem 20. Mai 2011 führte das RKI explorative Befragungen und verschiedene epidemiologische Studien durch, um die Ursache des Ausbruchs einzugrenzen und das Infektionsvehikel zu identifizieren.

Der zeitliche Verlauf des Ausbruchsgeschehens, die geografische und demografische Verteilung der Erkrankungsfälle sowie Informationen zu möglichen Expositionen, die bei der intensiven Befragung von zu Beginn des Ausbruchs erkrankten Patienten ermittelt wurden, deuteten auf ein mit EHEC kontaminiertes Lebensmittel als Infektionsvehikel hin. Bei den explorativen Befragungen wurden die Patienten mit einem ausführlichen Fragebogen zum Verzehr einer Vielzahl von tierischen und pflanzlichen Lebensmitteln einschließlich Sprossen befragt. Aufgrund der Befragungsergebnisse wurden Lebensmittel wie Rohmilch und rohes Fleisch, die häufig bei früheren

EHEC/HUS-Ausbrüchen eine Rolle spielten, bei diesem Ausbruch als nicht wahrscheinliche Infektionsvehikel angesehen.

Der Verzehr von Sprossen wurde nur von drei der zwölf am 20. / 21. Mai explorativ befragten 
Hamburger Patienten bejaht. Bei nachfolgenden epidemiologischen Studien wurden gemäß methodischen Forderungen und Standardvorgehensweisen (WHO 2008) nur die möglichen Expositionen im Fragebogen abgefragt, die potenziell in der Lage sind, epidemiologisch einen großen Teil des Ausbruchsgeschehens zu erklären.

Andernfalls erhöht sich bei Einschluss einer zu großen Anzahl von Expositionen die Gefahr fälschlich positiver statistischer Zusammenhänge (RKI 2011b).

Deshalb wurde in frühen Fall-Kontroll-Studien des RKI, bei denen erkrankte

Personen mit nicht erkrankten Personen hinsichtlich möglicher Expositionen verglichen werden, der Sprossenverzehr nicht erfasst. Bei diesen Studien ergaben epidemiologische Analysen, dass erkrankte Personen vor ihrer Erkrankung signifikant häufiger rohes Gemüse, speziell rohe Tomaten, Salatgurken und Blattsalat, gegessen hatten als nicht erkrankte Personen (RKI 2011b; Frank et al. 2011). Am 25. Mai 2011 wurde daraufhin von den Bundesbehörden BfR und RKI empfohlen, in Norddeutschland auf den Verzehr von Tomaten, Salatgurken und Blattsalat zu verzichten (http://www.rki.de/cln_178/nn_467482/DE/Content/InfAZ/E/EHEC/Gemeinsame_Stellungnahme_ RKI_BfR.html).

Die Üntersuchung des Auftretens von Erkrankungshäufungen nach dem Besuch zweier hessischer Firmenkantinen in Kooperation mit dem Amt für Gesundheit in Frankfurt und dem Kantinenbetreiber identifizierte Salat bzw. Bestandteile des an der Salatbar der Kantine verkauften Salats als wahrscheinliches Infektionsvehikel (RKI 2011b). Kunden, die in der Kantine Salat gekauft und mutmaßlich verzehrt hatten, hatten eine etwa sechsfach höhere Chance, blutigen Durchfall zu entwickeln als Personen, die dort keinen Salat gekauft hatten (Odds Ratio 5,8; 95 \% Konfidenzintervall (KI) 1,4-23,9; RKI 2011b).

In dieser Studie wurden die elektronischen Abrechnungsdokumente der Kantinenkunden ausgewertet, um unabhängig vom Erinnerungsvermögen der Erkrankten und Kontrollpersonen in Bezug auf ihren Lebensmittelverzehr mögliche Vehikel zu ermitteln. Eine ähnliche methodische Vorgehensweise wurde auch bei einer Restaurant-Kohortenstudie gewählt, die auf der Auswertung von detaillierten Zutatenlisten der Menüs basierte, die von Besuchern eines bestimmten Restaurants bestellt bzw. verzehrt worden waren (,,rezeptbasierte Restaurant-Kohortenstudie“; RKI 2011b). Hier wurden 10 Gruppen mit insgesamt 176 Teilnehmern identifiziert, die im Zeitraum zwischen dem 12. und 16. Mai in Restaurant $\mathrm{X}$ in Norddeutschland gespeist hatten und sich mit großer Wahrscheinlichkeit dort infiziert hatten. Von diesen Personen konnten 168 in die Datenanalyse eingeschlossen werden. Insgesamt waren 31 (18\%) Personen aus den Gruppen nach dem Restaurantbesuch an blutigem Durchfall erkrankt. Die Gruppenteilnehmer wurden befragt, welches Gericht sie bestellt hatten (auch mit Erinnerungshilfen durch Fotografien).

Die Angaben waren prinzipiell auch über die Buchungslisten des Restaurants bekannt, aus denen für die meisten Gruppen hervorging, welche Gerichte sie vorbestellt hatten. Zusätzlich wurde der Koch des Restaurants detailliert zur Zubereitung jedes Menüs, einschließlich der Mengenangaben für jede Zutat, befragt. Die Datenanalysen ergaben, dass Kunden, denen Sprossen serviert worden waren, ein etwa 14-fach höheres Risiko hatten, an blutigem Durchfall oder EHEC / HUS zu erkranken als Personen, denen keine Sprossen serviert worden waren (Relatives Risiko 14,2; 95 \% KI 2,6-?; RKI 2011b). Andere rohe Zutaten wie Tomaten, Gurken und Blattsalat standen in dieser Studie nicht mit einem erhöhten Erkrankungsrisiko in Zusammenhang (RKI 2011b). Allen erkrankten Personen war im Restaurant ein Gericht serviert worden, das Sprossen enthielt. Eine Mischung aus Bockshornklee-, Alfalfa-, Adzukibohnen- und Linsensprossen waren vom Restaurant X zur Garnierung von Salaten, auch von Beilagensalaten, verwendet worden.

Bei der Analyse der Lieferbeziehungen, die von der Task Force EHEC in Zusammenarbeit mit den Lebensmittelüberwachungsbehörden der Bundesländer durchgeführt wurde, konnte ermittelt werden, dass die Sprossen, die im Restaurant X serviert wurden, von dem Gartenbaubetrieb in Niedersachsen (Betrieb A) stammten, der auch mit anderen sogenannten Ausbruchsclustern in Verbindung gebracht werden konnte (Ergebnisbericht der Task Force EHEC 2011). 
Einen statistisch signifikanten Zusammenhang zwischen dem Verzehr von Sprossen und einer EHEC / HUS-Erkrankung lieferte auch eine weitere Fall-Kontroll-Studie (,,Rohkost-Fall-KontrollStudie"), die vom RKI durchgeführt wurde. Die Datenanalyse beruhte auf Angaben von 26 Fällen und 81 Kontrollpersonen.

Insgesamt gaben 6 von 24 (25\%) der Erkrankten und 7 von 80 (9\%) der Nichterkrankten (für die die entsprechenden Angaben vorlagen) an, Sprossen verzehrt zu haben. In multivariablen Analysen wurde für Personen, die Sprossen gegessen hatten, eine etwa vierfach höhere Erkrankungschance ermittelt als für Personen, die keine Sprossen gegessen hatten (Odds Ratio 4.4; $95 \%$ KI 1.1-18.0; RKI 2011b).

In weiteren drei Kohortenstudien wurden erkrankte und nicht erkrankte Teilnehmer von Reisegruppen, bei denen ein gemeinsamer Expositionsort, hier ein bestimmtes Restaurant, wahrscheinlich war, mit Hilfe von Menüplänen detailliert zu ihrem Lebensmittelverzehr befragt. Bei den ersten Auswertungen der Kohortenstudien ergab sich teilweise ein statistisch signifikanter Zusammenhang zwischen dem Verzehr einzelner Lebensmittel (v. a. von Salaten, Gurke und Tomate) und Erkrankung, jedoch gab es keine Mahlzeit bzw. kein Lebensmittel, was alle Erkrankungsfälle hätte erklären können (RKI 2011b). Retrospektiv, nach weiteren Befragungen der Küchenchefs, ließen sich allerdings alle Erkrankungsfälle über den Verzehr von Sprossen, die z. B. als Garnierung für bestimmte Gerichte verwendet worden waren, erklären. Die Analyse der Lieferbeziehungen konnte eine Verbindung zwischen den in den Kohortenstudien untersuchten Ausbruchsclustern und Sprossen aus Betrieb A und im Weiteren Sprossensamen aus Ägypten herstellen (Ergebnisbericht der Task Force EHEC 2011; EFSA 2011).

Basierend auf den Ergebnissen der epidemiologischen Studien des RKI und der Lieferkettenanalysen der Lebensmittelüberwachungsbehörden und der Task Force EHEC empfahlen BfR, BVL und RKI in einer gemeinsamen Pressemitteilung am 10. Juni 2011, bis auf weiteres Sprossen nicht roh zu verzehren (http://www.rki.de/cln 178/nn 467482/DE/Content/Service/

Presse/Pressemitteilungen/2011/08_2011.html). Diese Verzehrsempfehlung wurde am 21. Juli 2011 auf Sprossen aus ägyptischen Bockshornkleesamen eingeschränkt (http://www.rki.de/cln_178/nn_467482/DE/Content/Service/Presse/Pressemitteilungen/2011/10_ 2011.html).

Der Verzehr von Sprossen wurde von vielen erkrankten Personen bei den explorativen Befragungen offensichtlich nicht erinnert. Zum einen lag der Infektionszeitraum wegen der ungewöhnlich langen Inkubationszeit bei vielen befragten Patienten relativ weit zurück (10-14 Tage vor Beginn der Durchfallsymptomatik), so dass Detailfragen zum Lebensmittelverzehr nur schwer beantwortet werden konnten.

Zum anderen wurden Sprossen häufig nur als Garnierung, z. B. auf Beilagensalaten, verwendet, was die Erinnerung an den Verzehr zusätzlich erschwerte. So könnte auch erklärt werden, warum zwar Tomaten, Gurken und Blattsalat als typische Bestandteile eines gemischten Salats bzw. Salatbarbestandteile in den frühen epidemiologischen Studien als mögliche Infektionsvehikel identifiziert wurden, nicht aber Sprossen. Bei einer Wiederbefragung von Patienten und Kontrollpersonen nach Bekanntwerden des Zusammenhangs von Erkrankung und Sprossenverzehr, erinnerten sich drei von acht (37.5\%) Patienten an Sprossenverzehr, obwohl sie bei der Erstbefragung angegeben hatten, keine Sprossen gegessen zu haben oder sich nicht erinnern zu können. Von 37 wiederbefragten Kontrollpersonen änderte hingegen niemand seine ursprüngliche Aussage (RKI 2011b).

Einen überzeugenden Zusammenhang zwischen Sprossenverzehr und Erkrankung lieferten die rezeptbasierte Restaurant-Kohortenstudie und die Rohkost-Fall-Kontroll-Studie zusammen mit der Analyse der Lieferbeziehungen von sogenannten Ausbruchsclustern, die von der Task Force EHEC und den Lebensmittelüberwachungsbehörden der Bundesländer durchgeführt wurden. Die Analyse der Lieferbeziehungen von Ausbruchsclustern wird in einem Beitrag der Task Force EHEC in der vorliegenden Ausgabe dieses Journals näher beschrieben (Ergebnisbericht der Task Force EHEC 2011). 


\section{Zusammenarbeit zwischen Gesundheits- und Lebensmittelüberwachungs- behörden bei der Ausbruchsuntersuchung}

Eine enge Zusammenarbeit zwischen verschiedenen Institutionen und Behörden war für die zeitnahe Aufklärung des Ausbruchsgeschehens unerlässlich.

Dies betraf vor allem die Kooperation zwischen den Gesundheitsbehörden und den

Lebensmittelüberwachungsbehörden auf kommunaler, Landes- und Bundesebene.

Eine besonders enge Zusammenarbeit zwischen dem RKI und den

Lebensmittelüberwachungsbehörden der Länder und des Bundes erfolgte in der Task Force EHEC, die im Auftrag des BMELV vom BVL koordiniert wurde (Ergebnisbericht der Task Force EHEC 2011).

Hier wurden Erkrankungshäufungen, die in Zusammenhang mit bestimmten Expositionsorten wie z. B. Restaurants, Kantinen und Autobahnraststätten standen, hinsichtlich der Lieferwege und -ketten von Lebensmitteln analysiert, die an diesen Expositionsorten angeboten worden waren.

Ein wichtiger Beitrag der öffentlichen Gesundheitsbehörden war die Identifizierung und Priorisierung von wahrscheinlichen Expositionsorten, die Ausgangspunkte für die Lieferkettenanalysen waren. Informationen zu möglichen Expositionsorten, die das RKI von Ärzten, z. B. in Krankenhäusern, von Gesundheitsbehörden auf lokaler und Landesebene oder im Ausland, aus dem Surveillancesystem SurvNet und auch von Patienten selbst, z. B. über Befragungen, erhielt, wurden umgehend an das BfR, die Lebensmittelüberwachungsbehörden des betroffenen Bundeslandes und an die Task Force EHEC weitergeleitet, wenn es starke Hinweise darauf gab, dass die Exposition nur an diesem einen Ort erfolgt sein konnte.

Das war beispielsweise dann der Fall, wenn Mitglieder einer Reisegruppe mit mehreren Erkrankungsfällen vorher nur in einem bestimmten Restaurant gemeinsam gegessen hatten oder wenn bei Einzelfällen, die sich nur vorübergehend in Norddeutschland aufgehalten hatten, $z$. B. Touristen aus dem Ausland, nur ein einziger Expositionsort, z. B. eine Autobahnraststätte, in Frage kam (RKI 2011b). Auf diese Weise konnten RKI und die Task Force EHEC insgesamt 41 Ausbruchsorte identifizieren.

Alle untersuchten Ausbruchsorte hatten im betrachteten Zeitraum vor bzw. während des Ausbruchsgeschehens über mehrere Zwischenhändler Sprossen vom niedersächsischen Gartenbaubetrieb (Betrieb A) bezogen (Ergebnisbericht der Task Force EHEC 2011).

Als Infektionsvehikel im Ausbruchsgeschehen konnte letztlich mit sehr hoher Wahrscheinlichkeit Bockshornkleesamen identifiziert werden, der aus Ägypten in die Europäische Union importiert worden war, auch wenn ein belastbarer mikrobiologischer Nachweis des Ausbruchserregers O104:H4 in Samenproben der verdächtigten Charge(n) oder in Bockshornkleesprossen (oder anderen Sprossen) bisher nicht gelang (EFSA 2011).

Über eine verdächtigte Liefercharge von Bockshornkleesamen aus Ägypten konnten der Krankheitsausbruch in Deutschland und der HUS-Ausbruch in Frankreich im Juni 2011 in Zusammenhang gebracht werden (EFSA 2011).

In Bordeaux waren zwischen dem 15. und 20. Juni insgesamt 15 Personen wahrscheinlich durch den Verzehr von in Frankreich gezogenen Sprossen erkrankt (Gault et al. 2011).

Auch hatten in Niedersachsen mindestens zwei erkrankte Personen Sprossen aus einer Samenmischung gezüchtet und verzehrt, die unter anderem Bockshornkleesamen enthielt, der über mehrere Zwischenhändler auf die importierte verdächtigte Charge zurückgeführt werden konnte.

Die Untersuchungsergebnisse der europäischen Task Force, die von der EFSA koordiniert wurde (EFSA 2011), führten am 06. Juli 2011 zu einem Durchführungsbeschluss der Europäischen Kommission mit dem Ziel, verdächtige Bockshornkleesamenchargen vom Markt zu nehmen und zu vernichten (Amtsblatt der Europäischen Kommission 2011/402/EU). 


\section{Fazit}

Von Mai bis Juli 2011 ereignete sich in Deutschland der bisher größte Ausbruch von EHECErkrankungen. Bezogen auf die Anzahl der aufgetretenen HUS Fälle wurde auch weltweit bisher kein größeres derartiges Ausbruchsgeschehen beschrieben. Insgesamt erkrankten in Deutschland 855 Personen an HUS und 2.987 Personen an einer EHEC-Infektion, 53 Personen verstarben im Zusammenhang mit dem Ausbruchsgeschehen.

Es gibt derzeit keine Hinweise, dass sich nach Ende des Ausbruchs der Erreger EHEC O104:H4 endemisch in Deutschland etabliert hätte (RKI 2011b). Die Aktivitäten der epidemiologischen Überwachung werden weiter fortgesetzt und konzentrieren sich auf die Surveillance nach IfSG von EHEC-Infektionen und HUS sowie die Notaufnahmen-Surveillance zu blutigen Durchfällen in ausgewählten Krankenhäusern (RKI 2011b). Auch nach Ende des Ausbruchs gilt für Ärzte und den Öffentlichen Gesundheitsdienst eine erhöhte Wachsamkeit für das Auftreten von blutigen Durchfallerkrankungen und HUS einschließlich einer raschen Diagnostik mit Differenzierung bezüglich des Ausbruchstamms und Meldung in der ambulanten und stationären Versorgung (RKI 2011b). Sekundärinfektionen über eine Übertragung von Mensch zu Mensch sind weiterhin nicht auszuschließen.

Die Aufklärung des Ausbruchsgeschehens, vor allem die Identifizierung des Infektionsvehikels, war nur durch die enge Kooperation aller beteiligten Institutionen möglich.

\section{Danksagung}

Unser besonderer Dank gilt allen Patienten und Kontrollpersonen, Restaurant-, Kantinenbetreibern und Küchenchefs, die bereit waren, an Befragungen und epidemiologischen Studien des RKI teilzunehmen.

Weiterhin möchten wir uns bei den Ärzten und anderen Kollegen in den Krankenhäusern, die an Studien des RKI teilgenommen haben, sowie bei allen Gesundheitsämtern, Landesgesundheitsbehörden und Lebensmittelüberwachungsbehörden herzlich für die Zusammenarbeit bedanken. 


\section{Literatur}

Ackers ML, Mahon BE, Leahy E, Goode B, Damrow T, Hayes PS, Bibb WF, Rice DH, Barrett TJ, Hutwagner L, Griffin PM, Slutsker L (1998) An outbreak of Escherichia coli O157:H7 infections associated with leaf lettuce consumption. J Infect Dis 177:1588-1593

Amtsblatt der Europäischen Kommission (2011/402/EU): Durchführungsbeschluss vom 6. Juli 2011 über Sofortmaßnahmen hinsichtlich Bockshornkleesamen sowie bestimmter Samen und Bohnen aus ägypten; L179/10; 6. Juli 2011

Askar M, Faber MS, Frank C, Bernard H, Gilsdorf A, Fruth A, Prager R, Höhle M, Suess T, Wadl M, Krause G, Stark K, Werber D (2011) Update on the ongoing outbreak of haemolytic uraemic syndrome due to Shiga toxin-producing Escherichia coli (STEC) serotype O104, Germany, May 2011. Euro Surveill 16:pii=19883. Available online: http://www.eurosurveillance.org/ViewArticle.apsx?Articleld= 19883

Bell BP, Goldoft M, Griffin PM, Davis MA, Gordon DC, Tarr PI, Bartleson CA, Lewis JH, Barrett TJ, Wells JG et al (1994) A multistate outbreak of Escherichia coli O157:H7-associated bloody diarrhea and hemolytic uremic syndrome from hamburgers. The Washington experience. JAMA 272:13491353

Bielaszewska M, Mellmann A, Zhang W, Köck R, Fruth A, Bauwens A, Peters G, Karch H (2011) Characterisation of the Escherichia coli strain associated with an outbreak of haemolytic uraemic syndrome in Germany, 2011: a microbiological study. Lancet. doi:10.1016/S1473-3099(11)70165-7

Breuer T, Benkel DH, Shapiro RL, Hall WN, Winnett MM, Linn MJ, Neimann J, Barrett TJ, Dietrich S, Downes FP, Toney DM, Pearson JL, Rolka H, Slutsker L, Griffin PM, and the Investigation Team (2001) A multistate outbreak of Escherichia coli O157:H7 infections linked to alfalfa sprouts grown from contaminated seeds. Emerg Infect Dis 7:977-982

Centers for Diesease Control and Prevention (CDC) Outbreaks of Escherichia coli O157:H7 associated with petting zoos - North Carolina, Florida and Arizona, 2004 and 2005. MMWR Morb Mortal Wkly Rep 54:1277-1280

Die Task Force EHEC: Bernard H, Bisping M, Broschewitz B, Bucher M, Fetsch A, Förster D, Frandrup-Kuhr O, Fricke G, Greiner M, Gross S, Hänel C-M, Heusler K, Jähne J, Kenntner N, Kliemant A, Kühn K, Kutzke M, Ladehoff W, Luber P, Mosbach-Schulz O, Müller B, Rampp A, Reinecke A, Rosner B (2011) Ergebnisbericht der Task Force EHEC zur Aufklärung des EHEC O104:H4 Krankheitsausbruchs in Deutschland. Journal für Verbraucherschutz und Lebensmittelsicherheit, im Druck. doi:10.1007/s00003-011-0710-7

ECDC (2011) Shiga toxin-producing E. coli (STEC): Update on outbreak in the EU (27 July 2011, 11:00). http://www.ecdc.europa.eu/en/activities/sciadvice/Lists/ECDC\%20Reviews/ECDC_DispForm.aspx?Lis $\mathrm{t}=512 \mathrm{ff} 74 \mathrm{f}-77 \mathrm{~d} 4-4 \mathrm{ad} 8$-b6d6-bf0f23083f30\&ID=1166\&RootFolder=\%2Fen\%2Factivities\%2 Fsciadvice\%2FLists\%2FECDC\%20Reviews (Zugriff am 12.09.2011)

European Food Safety Authority (EFSA) (2011) Tracing seeds, in particular fenugreek (Trigonella foenum-graecum) seeds, in relation to the Shiga toxin-producing E. coli (STEC) O104:H4 2011 outbreaks in Germany and France. EFSA Frank C, Werber D, Cramer JP, Askar M, Faber M, an der Heiden M, Bernard H, Fruth A, Prager R, Spode A, Wadl M, Zoufaly A, Jordan S, Stark K, Krause G, for the HUS Investigation Team (2011) Epidemic profile of Shiga-toxin-producing Escherichia coli O104:H4 outbreak in Germany - Preliminary report. N Engl J Med. doi:10.1056/NEJMOa1106483

Gault G, Weill FX, Mariani-Kurkdijan P, Jourdan-da Silva N, King L, Aldabe B, Charron M, Ong N, Castor C, Macé M, Bingen E, Noël H, Vaillant V, Bone A, Vendrely B, Delmas Y, Combe C, Bercion R, d'Andingé E, Desjardin M, de Valk H, Rolland P (2011) Outbreak of haemolytic uraemic syndrome and bloody diarrhoea due to Escherichia coli O104:H4, south-west France, June 2011. Euro Surveill 16: pii=19905. Available online: http://www.eurosurveillance.org/ViewArticle.aspx?Articleld=19905 
Heymann DL (ed) (2008) Control of communicable diseases manual. 19th edn. American Public Health Association, Washington Keene WE, McAnulty JM, Hoesly FC, Williams LP Jr, Hedberg K, Oxman GL, Barrett TJ, Pfaller MA, Fleming DW et al (1994) A swimming-associated outbreak of hemorrhagic colitis caused by Escherichia coli O157:H7 and Shigella sonnei. N Engl J Med 331:579584

Keene WE, Hedberg K, Herriott DE, Hancock DD, McKay RW, Barrett TJ, Fleming DW (1997) A prolonged outbreak of Escherichia coli O157:H7 infections caused by commercially distributed raw milk. J Infect Dis 176:815-818

King LA, Mailles A, Mariani-Kurkdjian P, Vernozy-Rozand C, Montet MP, Grimont F, Pihier N, Devalk $\mathrm{H}$, Perret F, Bingen E, Espié E, Vaillant V (2009) Community-wide outbreak of Escherichia coli O157:H7 associated with consumption of frozen beef burgers. Epidemiol Infect 137:889-896

Michino H, Araki K, Minami S, Takaya S, Sakai N, Miyazaki M, Ono A, Yanagawa H (1999) Massive outbreak of Escherichia coli O157:H7 infection in schoolchildren in Sakai City, Japan, associated with consumption of white radish sprouts. Am J Epidemiol 150:787-796

RKI (2011a) Infektionsepidemiologisches Jahrbuch für 2010, Berlin

RKI (2011b) Bericht: Abschließende Darstellung und Bewertung der epidemiologischen Erkenntnisse im EHEC O104:H4 Ausbruch, Deutschland, Berlin

Tarr P, Gordon CA, Chandler WL (2005) Shiga-toxin-producing Escherichia coli and haemolytic uraemic syndrome. Lancet 365:1073-1086

Verma A, Bolton FJ, Fiefield D, Lamb P, Woloschin E, Smith N, McCann R (2007) An outbreak of E. coli 0157 associated with a swimming pool: an unusual vehicle of transmission. Epidemiol Infect 135:989-992

WHO (2008) Foodborne disease outbreaks: guidelines for investigation and control. http://www.who.int/foodsafety/publications/foodborne_disease/outbreak_guidelines.pdf)

WHO (2011) Outbreaks of E. coli O104:H4 infection: update 30, 22.07.2011.

http://www.euro.who.int/en/what-we-do/healthtopics/emergencies/international-healthregulations/news/news/2011/07/outbreaks-of-e.-coli-0104h4-infection-update-30

(Zugriff am 12.09.2011) 


\section{Abbildungen}

Abbildung 1 Epidemiologische Kurve der HUS- und EHECAusbruchsfälle (809 HUSund 2.717 EHECFälle mit bekanntem Erkrankungsbeginn an Durchfall im Ausbruchszeitraum) (Quelle: RKI 2011b).

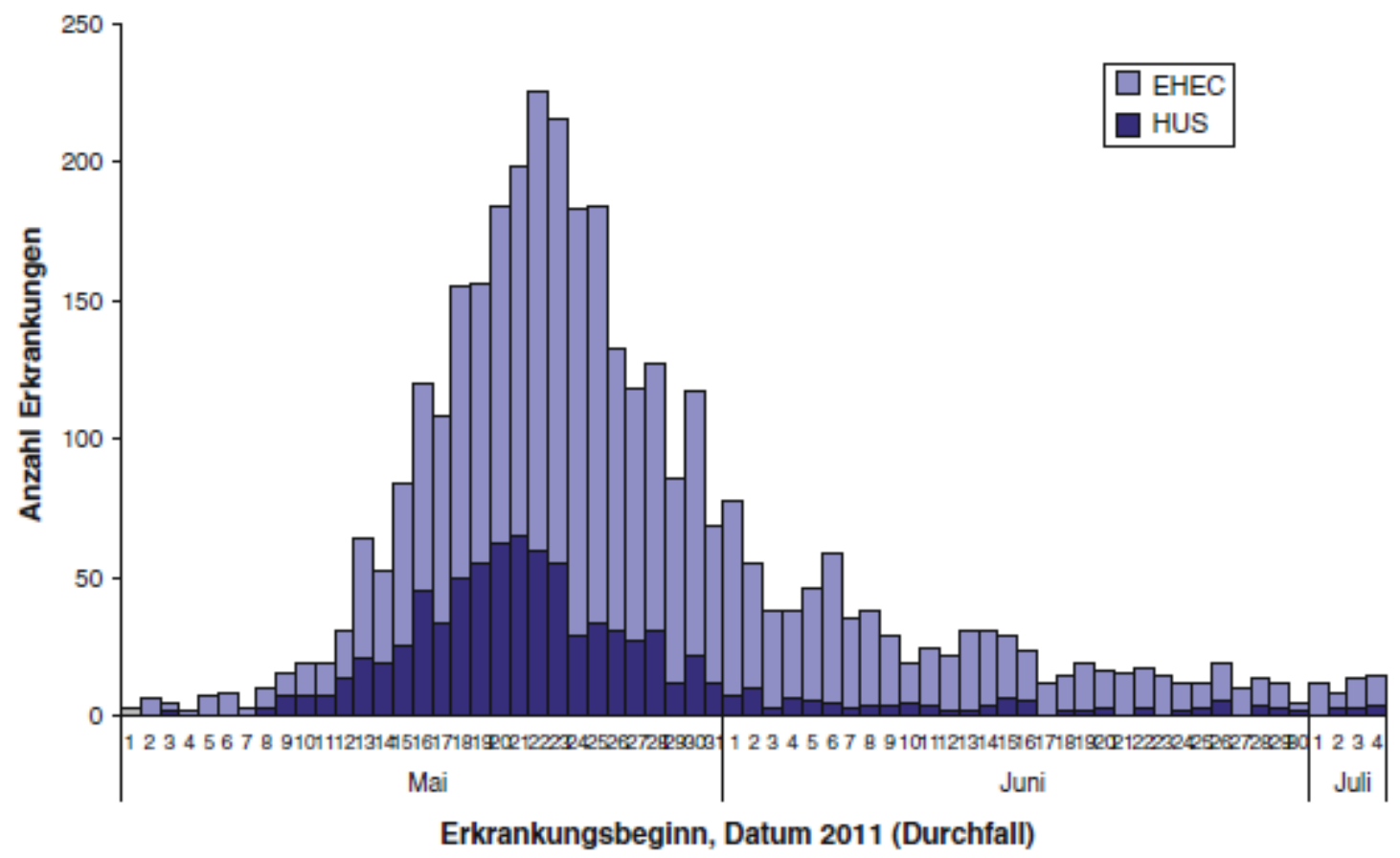


Abbildung 2 Inzidenz (Fälle pro 100.000 Einwohner) von HUS im Ausbruch, abgebildet nach Kreis, in dem die Infektion wahrscheinlich stattgefunden hat (Wohnortkreis oder, bei Reiseanamnese, Aufenthaltskreis zum Zeitpunkt der Infektion) (Quelle: RKI 2011b).

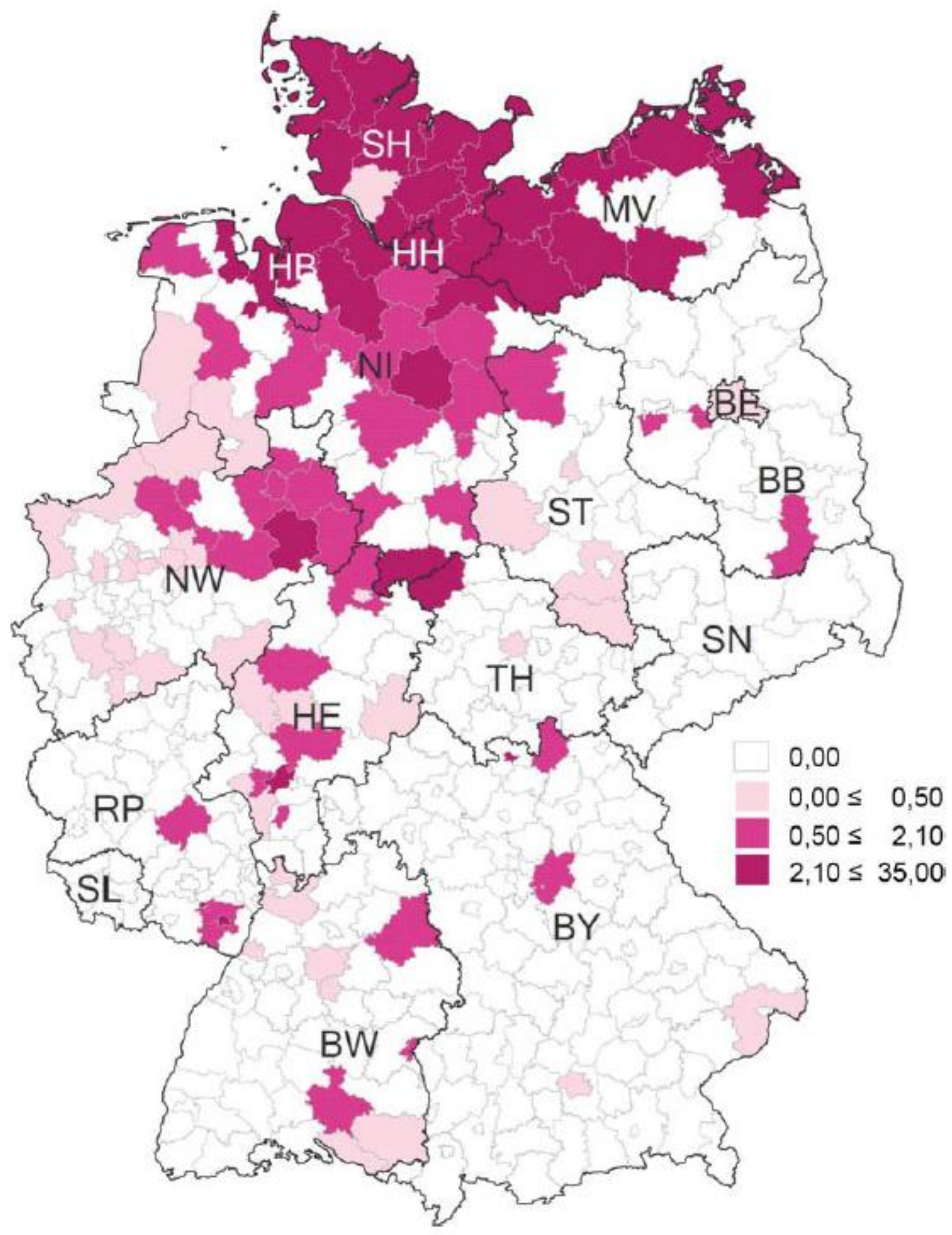

